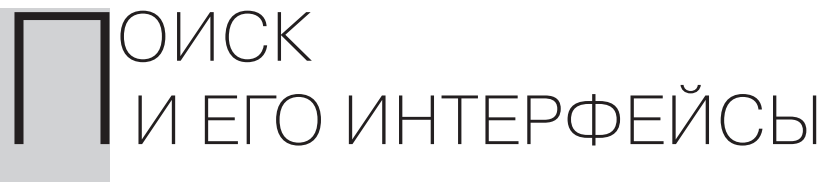

\title{
Илья Утехин
}

Илья Утехин - профессор факультета антропологии Европейского университета в Санкт-Петербурге. Адрес для переписки: ул. Гагаринская, 6/1, СанктПетербург, 191187, Россия. utekhin@yandex.ru.

За прошедшие три десятилетия информационные технологии революционизировали ту область повседневной деятельности, которую можно обозначить как управление личной информационной сферой. Ее составляют в совокупности систематически используемые пользователем информационные носители разных видов - как аналоговые (письменные и печатные), так и существующие лишь в цифровой форме. В основе интерфейса устройств, содержащих информационные объекты в цифровом виде (компьютер, смартфон), лежат аналогии тому, как люди обращаются с нецифровыми информационными объектами. Этнографические исследования выявили два полюса, к которым могут тяготеть стратегии организации информации: систематизация и «раскладывание по папкам», с одной стороны, и хронологическое складирование несистематизированных объектов - с другой. В интерфейсах цифровых сред воспроизводятся условия для обеих этих стратегий. При этом возможности оперирования цифровыми объектами ограничены тем, что заложено в дизайне интерфейса устройства и в дизайне взаимодействия с информационными сервисами. Хотя у цифровых объектов нет всех свойств материального предмета, они позволяют существенно расширить возможности поиска: здесь технологии позволяют эффективно находить объекты, вопрос о размещении которых нерелевантен, а технологии, чувствительные к контексту, позволяют производить поиск в цифровом информационном слое реального мира, откликаясь на запросы, целью которых является не узнать что-то, а удовлетворить ту или иную потребность, получив услугу. Наибольшее воздействие на повседневные практики поиска оказали технологии, опирающиеся на анализ поведения пользователя и обладающие агентностью: они предлагают пользователю такие объекты, о которых он не знал или которых он не искал.

Ключевые слова: информационный поиск; взаимодействие человека и компьютера; интерфейс; управление личной информационной сферой; агентность

Марка моего робота-пылесоса, NeatoBotvac (это не продакт-плейсмент, пылесос недавно опять сломался), напоминает названия первых больших компьютеров 1940-х годов: ENIAC, EDVAC, UNIVAC, но, хотя микропроцессоры этого пылесоса по своей производительности и сравнимы с этими ламповыми динозаврами, пылесос в целом скорее восходит к совсем другим существам той великой эпохи на заре кибернетики, к первым кибернетическим черепахам: тележкам на колесиках, которые перемещались по полу, следуя простому принципу - например, двигаясь всегда в сторону источника света. Любители самоделок могли без труда соорудить 
такое устройство в 1960-е годы при помощи фоторезистора, нескольких транзисторов и моторчика, а сегодня создать такого робота «Лего» из конструктора Lego Mindstorm может даже школьник младших классов. Подобно черепахе, которая в силу своего устройства (и программы) находит свет, пылесос обходит квартиру и собирает попавшийся ему мусор. Эти устройства действуют в реальном мире, перемещаются в окружающей их среде и вступают с ней во взаимодействие. Сказать, что черепаха «ищет» свет - вполне нормально, ее поведение целиком отвечает значению этого глагола; но если мы скажем, что пылесос «ищет» мусор, то тут, кажется, есть какая-то натяжка, связанная с тем, что привычное нам значение глагола «искать» предполагает целенаправленную деятельность со стороны кого-то, у кого имеется представление предмете поиска. То ли пылесосу мы еще не вполне готовы приписать полноценную субъектность, хотя он и ездит сам, то ли сомневаемся - справедливо - в его способности к представлениям о мире. Между тем по отношению ко многим компьютерным программам и сервисам, которыми мы пользуемся каждый день, мы привыкли употреблять глаголы «искать» и «найти», потому некоторые вещи без них нам было бы трудно или вовсе невозможно найти. Или это мы ищем? Но тогда что делают они?

Ниже мы попробуем взглянуть на всю сферу человеческой повседневной деятельности, которую можно назвать поиском, и отметить некоторые изменения, произошедшие в ней за последние десятилетия благодаря использованию цифровых технологий. Эти изменения в современной цивилизации носят революционный характер, трансформируя повседневные практики. Тем интереснее человеческое измерение информатики в нашей повседневности, то есть взгляд на поиск не как на проблему из области компьютерных наук, а как на вид человеческой деятельности, который включает в себя вынужденное общение с компьютерной технологией. Вопрос об интерфейсах здесь возникает потому, что технические устройства и системы, включенные в поиск у современного человека (от разных приложений в компьютере до смартфона или «умной» колонки Яндекса или Amazon), достаточно сложны и непрозрачны, чтобы обладать интерфейсом пользователя, то есть такой специально приспособленной частью, обращенной к пользователю, которая позволяет ему оперировать этой системой. Там, где интерфейс не стал еще само собой разумеющейся незаметной частью деятельности человека, он встает между пользователем и задачей, которую тот хочет решить, и требует внимания пользователя.

Это вообще касается пользовательских интерфейсов: нам часто приходится искать нужную функцию. В каком меню надо искать, чтобы сохранить презентацию в форме видеофайла? Есть ли вообще в этой версии PowerPoint эта функция? А где отключить вибрацию в телефоне? Ведь где-то это должно быть, но проекция возможных функций системы на доступные пользователю действия с органами управления бывает интуитивно не понятна. Проще и удобнее было бы сказать телефону «отключи вибрацию», и он бы понял и отключил. В этой мысли сегодня нет ничего странного - в том числе и в обращении к неодушевленному вроде бы предмету при помощи человеческой речи, как в сказочном управлении волшебным предметом. 
Удобство использования тех артефактов, которые предполагают работу с информацией, заключается в том, что они делают информацию наглядной и интуитивно понятной. Книги стоят рядами на полках корешками наружу, если их поддерживают «в порядке»; если книгу в библиотеке поставили «не туда», есть риск ее никогда не найти. На вахте ключи от помещений висят на доске с номерами, у каждого ключа есть своя бирка с номером, а каждый берущий ключ записывает сведения об этом событии в журнал, так что на доске сразу видно, какой ключ на руках, и по журналу должно быть понятно, кто его взял. Здесь порядок обеспечивает не только дизайн, но и упорядоченное правилами поведение участников ситуации. Если взял ключ и не записал - это непорядок, и вахтер обязан был проследить; в отсутствие записи остается надеяться на то, что вахтер вспомнит, кто взял этот ключ. Порядок, в перспективе обозначенной проблематики, - это такая организация вещей и поведения, которая облегчает поиск. Порядок в быту означает, что вещи на предсказуемых местах (и даже мусор и отходы именно там, где им положено быть - в мусорном ведре, а не там, где бы они воспринимались как грязь), так что бытовой порядок - тоже инфраструктура поиска: в упорядоченном жилище меньше времени уходит на поиск носков и очков, а чистые и грязные носки лежат в разных местах.

Устройство повседневной вещной среды как инфраструктура поиска пока что никак не зависит от цифровой технологии. Но поиск не предмета, а информации сегодня зачастую опирается на цифровые системы: значительная часть наших поисков производятся с использованием компьютера и мобильного телефона. Можно попробовать провести эксперимент методом систематического самонаблюдения или же сделать мысленный эксперимент: отмечать в течение некоторого времени все действия, которые можно отнести к поиску, - начиная с самого утра, от момента нащупывания телефона, чтобы отключить будильник, от поиска места для парковки, подарка для ребенка-именинника и до мгновенного решения, куда бы положить использованный чайный пакетик.

Если присмотреться к получившемуся списку и попробовать выделить попавшие сюда типы действий, вынеся за скобки все различия между предметами и способами поиска, то получится несколько ситуаций, которые задают ориентиры в пространстве возможностей:

1. Я точно знаю, что я ищу, и знаю, где оно находится, и сейчас найду (например, перебирая бумаги в папке, перелистывая страницы в книге, просматривая список контактов).

2. Я точно знаю, что я ищу, но не знаю, где оно. Как только я увижу искомое, я сразу его узнаю (в том числе это ситуации, которые описываются как «я потерял X», «кто-то куда-то засунул X»; но это и движение вдоль ряда припаркованных автомобилей в поисках свободного места, и поход за грибами в лес: какие грибы попадутся, я не знаю, но предполагаю, что здесь могут быть грибы, тут даже пахнет грибами). 
3. Я примерно знаю, что нужно, но не знаю точной формулировки и названия, и не знаю точно, где я это найду. Увижу - оценю, годится это или нет. Это ситуация, когда я ищу что-то, чтобы подарить другу на день рождения, и когда я не знаю, куда пойти сегодня поужинать.

Это не исчерпывающий список типов ситуаций, но уже и этого различения достаточно, чтобы указать на то, что цифровые технологии в поиске революционизируют ситуации третьего типа и выручают нас в случаях, когда неопределенность касается не только места, но и собственно объекта поиска. Если бы мы в наблюдении жизненных ситуаций оттолкнулись не от значения глагола «искать», а от значения глагола «найти», то этот пласт нечаянных находок оказался бы более явным. Ведь «найти» - это не только случаи типа «искал - нашел» (когда знал, что́ искал), но и типа «шел-шел и нашел», то есть нечаянно обнаружил что-то интересное. Или когда кто-то нам посоветовал или навел на мысль.

Более того, эти базовые ситуации отражают две стратегии поиска, для которых в английском языке есть два разных глагола - search и browse - и которые связаны с организацией среды, где производится поиск. Так, если книги в библиотеке расставлены по размерам и алфавиту, то ходить вдоль полок - занятие малоосмысленное, если у вас нет точного шифра книги; напротив, если используется десятичная классификация Дьюи, то открытые фонды дают читателю доступ к систематически упорядоченной расстановке книг по темам, и тогда после того, как читатель нашел интересующий его тематический раздел, он перебирает стоящие на этих полках книги с большой пользой для себя, потому что может найти что-то, что ему интересно, но о существовании чего он не подозревал - а потому и не мог искать этого целенаправленно.

Вещи даже в аккуратном жилище не могут быть расставлены с такой же систематичностью, как в библиотеке: слишком они разные. Во введении к своей книге «Living with Complexity» классик в области когнитивных аспектов дизайна Дональд Норман поместил фотографию рабочего кабинета американского общественного деятеля Эла Гора, на которой мы видим кроме самого хозяина кабинета, сидящего за столом с тремя компьютерными мониторами, несколько поверхностей, заваленных бумагами; точнее, это стопки бумаг, книг и папок (Norman 2010). Знакомая в сущности картина, ведь не только у Эла Гора на столе царит то, что ассоциируется с творческим беспорядком. Норман утверждает, что расположение этих стопок и бумаг в стопках отражает ценную информацию, организующую этот своеобразный порядок в глазах его пользователя (и творца): наведение в таком кабинете «порядка» могло бы оказаться губительно для работы.

Заваленные бумагами столы как феномен персональной информационной среды привлекали внимание исследователей, начиная с работы Томаса Мэлоуна (Malone 1983), где автор задается вопросом: что люди делают с бумажными документами и как складывают их в папки у себя на рабочем месте и не только. Мэлоун наблюдает как аккуратистов, так и людей с заваленными бумагами столами, находя этим привычкам объяснение и выделяя единицы организации информации. Аккуратисты стремятся каждый новый документ приписать определенной 
категории и помещают его в соответствующую папку или лоток, но у любого такого места обозначено название категории документов, сюда попадающих. Тогда как хозяева рабочих мест, содержащихся в «беспорядке», используют другую стратегию: по мере поступления новых документов на их столе растут стопки, при этом содержание каждой стопки - неподписанной - менее структурировано: здесь оказываются объекты разного рода, разложенные либо в хронологическом порядке (свежее сверху), либо в порядке важности и срочности (сверху самое срочное). Эти стратегии в разной степени характерны для разных видов деятельности. Те, чья работа предполагает стандартные процедуры обработки ограниченного круга документов - бухгалтеры, кадровики, продажники, - могут разбить этот поток на категории. А у представителей более творческих специальностей больше таких бумаг, которые не поддаются классифицированию.

Важный вывод, к которому приходит Мэлоун, состоит в том, что папки и стопки оказываются элементарными кирпичиками, методами управления бумажными документами, и они дополняют друг друга: в папке или лотке, которые определяют категорию содержимого, может находиться стопка документов. Тут впору задуматься, что не только папки, но и пластиковые файлики, скрепки и степлеры оказываются инструментами организации информации.

Область, к которой относятся исследования Мэлоуна, - это «управление личной информационной сферой» (personal information management). Она возникла на пересечении нескольких дисциплин для того, чтобы решать практические вопросы, связанные с разработкой информационных сервисов. Одна из самых заметных публикаций (Jones and Teevan 2007), являющаяся результатом совместного проекта исследователей из Microsoft, IBM, Google и Palo Alto Research Center, отражает потребности индустрии в понимании повседневного мира пользователя информационных технологий, отталкиваясь от этнографического описания того, как люди в реальной жизни реализуют свои потребности. Этот и подобные проекты сделали вклад в создание тех систем поиска, которые доступны нам сегодня. Ниже я буду ссылаться не только на исследования, но и на наблюдения за своей повседневностью, которым читатель без труда найдет параллель в собственной практике.

\section{СОЗДАТЬ, НАЗВАТЬ, ПОМЕСТИТЬ, НАЙТИ}

Наблюдения за порядком и его использованием в разных контекстах дома и в офисе подталкивают к тому, чтобы увидеть цифровые и обычные артефакты-носители информации как составляющие персонального информационного пространства. Человек их организует: получает или создает, сохраняет, называет, снова находит. Сюда входят книги и бумаги, электронные письма, файлы в разных компьютерах и в облачных сервисах, ссылки и закладки в браузерах. Собственно, поскольку теперь документы и данные не только в компьютере, но и в облаке, на внешних дисках, на флеш-накопителях, вся совокупность информации, которую человек для себя организует, распределена между разными местами. Внутри этой совокупности информационных артефактов пользователь создает более-менее 
организованные островки, связанные с той или иной темой или проектом (Jones and Teevan 2007).

Особенность цифровых документов в том, что это объекты, у которых нет физического воплощения, пока мы их не распечатали. На «рабочем столе» в компьютере, где «папка» - это элемент интерфейса в компьютере, а документы в ней - это файлы, мы можем увидеть только то, что придумал дизайнер, чтобы отобразить эти абстрактные объекты: в отличие от физического объекта, у которого его видимые свойства и возможности действия с ним - это само собой разумеющийся его аспект, для виртуального объекта все видимые характеристики зависят от замысла дизайнера, создавшего внешние проявления информационным структурам, которые сами по себе невидимы и вообще недоступны восприятию. Дизайнер должен был догадаться отразить разницу между пустой папкой и папкой, где что-то есть, во внешнем виде иконки папки, иначе мы этой разницы не увидим. В результате абстрактные объекты (файлы, соединения, серверы, учетные записи, сеансы и т.п.) в метафорическом мире интерфейса отображены таким образом, который определяет, что с ними можно сделать.

В привычном нам графическом интерфейсе есть пространственное расположение объектов, которое может нам подсказывать, где что искать. Тут есть аналог не только папок, но и стопок. Цифровые стопки - это документы внутри неорганизованной папки или прямо сваленные на десктоп, но одновременно десктоп является еще и аналогом папки в файловом навигаторе. Наученный горьким опытом, я знаю, что чем больше тяжелых файлов на декстопе, тем компьютеру тяжелее. Лучше сваливать файлы в готовые большие папки, они уже заданы в системе: «Документы», «Музыка», «Видео». Уже беглый взгляд в мой компьютер показывает, что самая большая свалка у меня в разделе «Загрузки». Но пока мне удается решать большинство задач без наведения порядка. Меня выручает текстовый поиск по содержанию документов, довольно недавнее нововведение; но с нетекстовыми документами (картинками, аудио- и видеофайлами) все сложнее. Особенно обидно не найти что-то такое, что у тебя уже где-то точно есть ${ }^{1}$. В то же время если проще без труда найти заново в сети, то создается ситуация, когда в компьютере оказывается несколько копий одного и того же документа.

Организация информации в компьютере предполагает несколько типов действий. Во-первых, требуется создать те самые единицы организации - папки. Вовторых, файл или папку надо назвать и, в-третьих, куда-то поместить. Продуктивно было бы понаблюдать, как мы называем и куда помещаем файлы, и попытаться в каждом случае сформулировать, почему мы делаем именно так. Снабжая файлы (например, фотографии) названиями, раскладывая их по папкам и называя папки, пользователь фактически задает онтологию, диктует устройство этого фрагмента мира и потом опирается в процессе поиска глазами на свое представление о том, как этот фрагмент устроен.

1 Отдельный исследовательский проект был посвящен тому, как люди сохраняют найденное, имея в виду дальнейшее использование (Jones 2008). 
Показательны сбои, когда названия файлов и папок недостаточно информативны для того, чтобы ими пользоваться. Вот студенты сдают преподавателю работы, загружая файлы, где в названиях нет их фамилии. Вот на рабочем столе компьютера в зале, где проходит конференция, файл, озаглавленный «моя презентация.pptx». Названия бывают рассчитаны только на самого автора. Зачастую наша деятельность по категоризации единиц информации минимально достаточна для данного момента и опирается на сиюминутные факторы, а не на осознанную нужду в создании метаинформации, на которую потом можно опереться при поиске.

Если в системе по умолчанию есть папки для музыки, видео, фотографий, документов, то в свою папку, которая посвящена, например, материалам к лекции по определенной теме, я помещаю и тексты статей, и книги, и картинки, и видео, иногда и звуковые файлы, и презентации, которые к этой теме имеют отношение. Там внутри все лежит грудой, но можно было бы при желании навести порядок и сложить по типам файлов. Как указывает Марк Лансдейл (Lansdale 1988:56), складывание в груду или стопку, которое Мэлоун противопоставил раскладыванию по папкам, представляет собой компромисс, когда классификация оказалась бы слишком сложна: чтобы избежать необходимости классифицировать, можно сложить в одном и том же месте.

Нередко приходится искать одно и то же, хорошо знакомое, например, пароли. Браузер помогает нам, запоминая пароли, используемые в интернете, а есть и специальные сервисы, которые берутся хранить все наши пароли. На рубеже веков бумажки с паролем для входа в систему часто прикрепляли к монитору компьютера. В наши дни мне приходится заходить в мессенджер, в чате с одним из собеседников смотреть галерею отправленных фотографий и находить среди них ту бумажку с написанным на ней паролем, которая однажды была сфотографирована на телефон моим собеседником и отправлена мне в этом чате.

Тут показательно использование средства коммуникации (электронной почты или мессенждера) для хранения информации. Использование электронной почты для того, чтобы отправить документ самому себе, было подмечено давно (Jones, Bruce, and Dumais 2001), и реакцией на такое использование были возможности, введенные в интерфейс почтовых клиентов для раскладывания сообщений по папкам и для эффективного поиска. Эффективный поиск отчасти избавляет от необходимости прикладывать усилия к организации информации (Cutrell, Dumais, and Teevan 2006). Сегодняшний почтовый ящик это еще и место хранения информации, где поиск позволяет находить все, что встречалось в переписке, и при этом ящик не надо чистить, потенциально он бесконечно велик.

Авторы исследований поиска информации в информационных системах отмечали, что люди предпочитали ориентироваться в расположении искомой информации, находить ее, отталкиваясь от контекста, а не «телепортироваться» непосредственно к результату (Teevan, Capra, and Pérez-Quiñones 2007:26). Так, если мы ищем в компьютере нужный файл, содержание которого мы помним, но название забыли, мы можем в принципе воспользоваться поиском, который выдаст нам все файлы, где встречается некоторое слово, которое, как мы знаем, точно встре- 
чается в этом файле и едва ли встречается во многих других. Это достаточно эффективно, потому что все файлы в современных операционных системах проиндексированы. Но другой, более человеческий, вариант заключается в том, чтобы просмотреть подряд названия всех файлов в той папке, где, как мы предполагаем, может лежать искомое. Узнать среди многих сообщений электронной почты от конкретного отправителя нужное сообщение проще, чем придумать ключевое слово и осуществить поиск по нему. И это несмотря на то, что сегодня эффективный поиск в MacOS и в почтовых ящиках типа Gmail приучает нас к тому, что поиск по ключевому слову совсем не сложнее (Jones, Wenning, and Bruce 2014).

\section{НЕЗАМЕТНАЯ РАБОТА И МИНИМАЛЬНОЕ УСИЛИЕ}

Предположим, мы что-то искали в интернете - и много чего нашли, причем значительно больше, чем можно просмотреть и освоить за один раз. Открывая вкладку и не закрывая ее, оставляя ее «на потом», мы не то чтобы планируем свою будущую деятельность (вполне возможно, что мы никогда не прочитаем содержимое этой вкладки), но отмечаем важность найденного и создаем возможность к нему вернуться. Вот когда несколько вкладок (иногда десяток) открыты в каждом из нескольких окон моего браузера - это тоже полезный, хотя и несовершенный способ организации информации. Браузер иногда обрушивается под весом этих открытых окон и вкладок, и, открывая его в следующий раз, я снимаю галочки с тех вкладок, которые уже перестали быть актуальными. Эти действия отражают оценку актуальности того, что было как бы отодвинуто на заднюю конфорку, чтобы потом к этому обратиться. Когда потом захочешь заново это найти, не всегда получается. Обычность таких обстоятельств заставляет задуматься о том, что значительная часть файлов в компьютере, страниц в вебе и всего того, что мы когда-то посетили или себе сохранили куда-то в форме ссылки или целого документа, никогда не будет востребована. То есть тысячи книг и статей в формате pdf в моем ноутбуке я никогда не прочитаю, хотя мне в какой-то момент показалось важным, чтобы они у меня были. Значительная часть из них никогда не будет востребована по той причине, что они не были сохранены в понятные и прозрачные места и не были названы понятно. Такой документ я могу найти не целенаправленно, а разве что случайно, воспользовавшись функцией текстового поиска и запрашивая документы, содержащие те или иные термины.

Если есть все время растущий - от одного моего компьютера к другому и на все увеличивающемся числе съемных носителей информации - объем памяти, то почему бы не хранить больше того, что мне необходимо? Если бы на всех флешнакопителях, которые где-то теряются, была только остро необходимая информация в одном экземпляре, жизнь была бы гораздо сложнее.

Я обратил внимание на то, что, хотя и делаю иногда закладки в браузере, практически никогда ими не пользуюсь потом и не трачу усилия на то, чтобы эти закладки организовать. Их хронологическое расположение в списке очень характерно, поэтому использование элементов цифровых эквивалентов стопки может опираться на последовательность элементов в ней. 
Например, такой стопкой является список звонков в смартфоне. Если я по той или иной причине не сохранил в списке контактов номер входящего звонка с незнакомого телефона, я тем не менее могу воспользоваться этим номером, вспомнив, что водопроводчик звонил мне вчера вечером и вот это должен быть его номер. Как отмечает Ян Чипчейз, в свое время реализовавший в исследовательском центре Nokia несколько крупных этнографических проектов, связанных с мобильностью и дизайном мобильной технологии для неграмотного человека (а таких пользователей мобильной связи около миллиарда), история звонков - самый простой и удобный заменитель списка контактов (Chipchase 2008:85). С начала XXI века дизайнеры пытались сделать доступным в том числе и неграмотному пользователю не только мобильную связь, но и интернет, и сразу же голосовые интерфейсы стали рассматриваться как важнейшее средство, несмотря на ограничения голосовой технологии.

В начале 1990-х в знаменитой статье Марка Уайзера (Weiser 1991) было представлено видение будущего, когда компьютерная технология срастется с окружающим повседневным миром подобно тому, как созрела и сделалась незаметной технология письма. Буквы у нас везде, но не требуют особенного внимания: информация растворена повсюду, надо только уметь читать. Это как дорожные знаки для водителя: когда он видит дорожный знак, он не производит сознательный акт интерпретации. Технология исчезает на периферии внимания (Norman 1998). Визионеры этого сравнительно недавнего прошлого придумали «умные среды» вроде «умного дома», но довольно неожиданно оказалось, что в начале XXI века главной платформой для технологий поиска окажется смартфон - мобильное устройство, наделенное сенсорами и чувствительное к контексту. Чаще всего мы что-то ищем сегодня при помощи мобильного устройства.

\section{ПРИПИСЫВАНИЕ НАМЕРЕНИЙ И АГЕНТНОСТЬ ИНСТРУМЕНТА}

За последние четверть века поисковые сервисы дважды пережили революционные перемены, которые на наших глазах резко улучшили качество поиска и сделали поиск таким, каким мы его видим сейчас². Выделяются три отчетливых этапа в эволюции технологии поиска (Baeza-Yates, Broder, and Maarek 2011). Изначально поиск производился по проиндексированным коллекциям текстовых документов на предмет вхождения термина или терминов запроса в документ, причем могла учитываться частотность и специфичность терминов запроса, а совпадение со словами в заголовке или в списке ключевых слов, указанных в метаданных документа, оценивалось как более весомое. Поисковые системы вроде «Альтависты» работали небыстро, растущий размер сети представлял для таких систем проблему, а поисковая выдача была заспамлена нерелевантными ссылками. Начиная с 1998 года Google и другие поисковые системы начинают опираться не только на

${ }^{2}$ См. историю идеологии и технологий Google в популярном изложении (Levy 2011), а также попытку культурологического осмысления поисковых технологий в книге (Hillis, Petit, and Jarrett 2013). 
индекс отдельных документов, но и на анализ структуры сети, на «ссылочную релевантность»: авторитетность и релевантность страницы определяется с учетом данных о том, какие другие страницы ссылаются на эту страницу (своеобразный эквивалент «индекса цитирования»). Соответственно, чем больше веб и чем больше ссылок можно учесть, тем лучше получаются результаты.

Следующий этап, продолжающийся и сегодня, связан с учетом поведения пользователей и внетекстовых факторов. Как пользователи формулируют и переформулируют запрос, по каким ссылкам на странице результатов они переходят, что этот конкретный пользователь уже искал и что он нашел - собирая эти и подобные данные и опираясь на контекст (от времени суток до данных о местонахождении пользователя), поисковая система стремится разглядеть потребность пользователя, которая стоит за поисковым запросом, и уже в ходе формулирования запроса предлагает коррекцию опечаток и варианты автоматического дополнения запроса, а на странице результатов выделяет разные типы информации - не только текстовые страницы, но и изображения, видео, книги, статьи в «Википедии». Получается, что поисковый сервис как бы читает мысли пользователя и даже предлагает ему что-то такое, что тот не называл в своем запросе, подобно тому, как во многих сервисах встроены рекомендации (если вы купили эту книгу, вам могут понравиться и другие, которые купили те пользователи, которые купили такую же). Пользователь, получается, в обмен на поток данных о своем поведении получает от системы более качественные результаты. Заодно он позволяет системе использовать себя как потребителя контекстной рекламы, на чем и строится поисковый бизнес.

Отталкиваясь от данных о действиях пользователя, алгоритмы ранжирования корректируют выдачу под персональные нужды этого пользователя и определяют, чего он хочет. Традиционно выделяют три измерения, к которым тяготеют поисковые запросы (см., например: Broder 2002) - информационные, навигационные и транзакционные. Информационные запросы касаются не только теоретических вопросов и сведений, в том числе для решения кроссвордов ([царевич военный вождь троянцев], [полкласса как пишется], [суть идеи синергетики]), но и решения конкретных задач ([песочное тесто рецепт]). Навигационные запросы имеют в виду навигацию и в сети, то есть связаны с поиском сайтов разных организаций и сервисов, и в реальном мире, когда пользователя интересуют адреса и схемы проезда. За транзакционными запросами пользователя стоит намерение произвести конкретное действие - купить билет или товар, заказать пиццу, скачать файл. По отношению ко многим запросам, особенно коротким, нельзя однозначно определить это действие (скажем, тот, кто вводит в «Яндекс» [свадебные платья], может хотеть купить, взять напрокат или просто посмотреть модели). Система должна такой запрос расширить и проинтерпретировать.

Пользовательский интерфейс поискового сервиса может выглядеть как поле, куда мы вводим текст, или как диалоговая система вроде виртуальных ассистентов Siri или Алисы, голос которых доносится из смартфона или специальной колонки. Сама поисковая система оказывается теперь своеобразным интерфейсом между пользователем и реальностью. При этом отношение между мной и содер- 
жимым заваленного бумагами стола не такое, как между мной и миром, в котором я ориентируюсь и к которому получаю доступ через «Яндекс» или Google. Разница в степени агентности интерфейса. Как на лифте нельзя приехать на этаж, которому не соответствует никакая кнопка из панели управления, так и из поисковой системы нельзя выудить результат, который не проиндексирован или удален из выдачи. А если фильтрация информации не осознается и поисковая выдача воспринимается как прямой доступ к реальности, не подменяет ли карта территорию? Ведь это поисковая система принимает решение, через логику, заложенную в ее алгоритмах, включать ли в выдачу нежелательную для кого-то или для чего-то информацию (например, нарушающую тот или иной закон). B 2019 году Google, как и в континентальном Китае, подчинился местным регуляторам в России и перестал показывать в выдаче страницы, к которым в этой стране ограничен доступ, - и это не только ресурсы, предоставляющие доступ к пиратскому контенту или ссылки на средства обхода блокировок.

По отношению к какой реальности оказывается интерфейсом поисковый сервис? Сегодня пользователь ищет не только текстовые документы в вебе, но и что-то в реальном мире: найти, где поужинать; найти дорогу по карте и объехать пробки; увидеть, как выглядит то место, куда мы едем; найти партнера для игры и партнера на сайте знакомств. И так вплоть до придания смысла фрагменту окружающего мира, когда он дает специальному сервису послушать через смартфон кусочек песни, чтобы определить, что это за произведение. То есть интерфейс поиска мне предоставляет чувствительный к локации и к другим контекстуальным обстоятельствам мобильный гаджет (под это определение подходит, в частности, современный автомобиль). И сама по себе эта осведомленность о контексте создает условия для того, чтобы предложить мне, куда бы еще пойти, что бы еще купить. Чего бы еще возжелать, что тут еще есть поблизости?

Представим себе такой сервис с рекомендательным функционалом (и с учетом истории поисков и действий пользователя) в виде автономного интеллектуального ассистента, к которому пользователь обращается всякий раз, когда у него возникает желание, например, послушать музыку. Такой ассистент может расти вместе с ребенком, сначала рекомендуя ему детский репертуар, потом руководя его более зрелым медиапотреблением. Обладая памятью и осведомленностью о вкусах и привычках человека лучше него самого и его родителей, такой ассистент превратился бы в вынесенную наружу часть пользовательского бессознательного, стирая грань между желанием и рекомендацией: он лучше меня знает, чего мне сейчас захотелось.

Визионеры рубежа веков видели будущее, опираясь на представление о «семантическом вебе» и интеллектуальных агентах, примерно так: многие функции возьмут на себя интеллектуальные ассистенты, в том числе с речевым интерфейсом (Berners-Lee, Hendler, and Lassila 2001; см. также, например: гл. 9 в Marchionini 1995). Скажем, если надо записаться к врачу, то твой виртуальный агент выспросит у тебя всю необходимую информацию и организует визит, посылая запросы агентам врачей и агентам твоей страховой компании, пользуюсь богато размеченными данными семантического веба. Это уже не просто поиск, а сложные (хотя и 
стандартные) действия по поиску и согласованию, которые обычно производит сам человек, но которые готов взять на себя наделенный искусственным интеллектом ассистент. Сегодня нетрудно вообразить себе такой навык яндексовской Алисы: для его создания доступны все необходимые технологии.

Такое вынесение функций наружу, когда поиск и организация информации опираются на использование сущностей, обладающих способностью к самостоятельному поведению и к общению с человеком на его человеческом языке, - нечто существенно новое в тренде развития интерфейсов, которые на каждом шаге включают все больше и больше собственно человеческих способностей (Утехин 2012). Своей самостоятельностью такие агенты напоминают роботический пылесос, но ни он, ни современные ассистенты с речевым интерфейсом не стали само собой разумеющейся частью повседневности во многом в силу того, что технологическое мифотворчество - важнейший аспект культуры потребления технологических новинок: благодаря этому соусу люди готовы попробовать воспользоваться весьма незрелой технологией уже хотя бы потому, что она отвечает мечтам о будущем.

Между тем, чтобы почувствовать масштаб революции, внутри которой мы находимся, достаточно вообразить себе, как выглядел бы тридцать лет назад предложенный в начале этого текста мысленный эксперимент (осознать и зафиксировать за день все действия, которые можно обозначить глаголами «искать» и «найти»). В рутинную повседневность вошел не только глагол «погуглить», но и способность практически не отрываясь от чтения или беседы погуглить - и найти.

\section{СПИСОК ЛИТЕРАТУРЫ}

Утехин, Илья. 2012. «Взаимодействие с “умными вещами": введение в проблематику». Антропологический форум 17:134-156.

Baeza-Yates, Ricardo, Andrei Z. Broder, and Yoelle Maarek. 2011. “The New Frontier of Web Search Technology: Seven Challenges." Pp. 3-9 in Search Computing: Trends and Developments, edited by Stefano Ceri and Marco Brambilla. New York: Springer.

Berners-Lee, Tim, James Hendler, and Ora Lassila. 2001. "The Semantic Web." Scientific American, May, 28-37.

Broder, Andrei Z. 2002. "A Taxonomy of Web Search." ACM SIGIR Forum 36(2):3-10.

Chipchase, Jan. 2008. “Reducing Illiteracy as a Barrier to Mobile Communication." Pp. 79-89 in Handbook of Mobile Communication Studies, edited by James E. Katz. Cambridge, MA: MIT Press.

Cutrell, Edward, Susan Dumais, and Jaime Teevan. 2006. "Searching to Eliminate Personal Information Management." Communications of ACM 49(1):58-64.

Hillis, Ken, Michael Petit, and Kylie Jarrett. 2013. Google and the Culture of Search. London: Routledge. Jones, William. 2008. Keeping Found Things Found: The Study and Practice of Personal Information Management. Burlington, MA: Morgan Kaufmann Publishers.

Jones, William, Harry Bruce, and Susan Dumais. 2001. "Keeping Found Things Found on the Web." Pp. 119-126 in Proceedings of the Tenth International Conference on Information and Knowledge Management. Atlanta, GA: ACM.

Jones, William, and Jaime Teevan, eds. 2007. Personal Information Management. Seattle: University of Washington Press.

Jones, William, Abe Wenning, and Harry Bruce. 2014. “How Do People Re-Find Files, Emails and Web Pages?" Pp. 552-564 in Breaking Down Walls: Culture, Context, Computing, edited by Maxi Kindling and Elke Greifeneder. Proceedings of iConference 2014, Berlin, March 4-7, 2014. http:// hdl.handle.net/2142/47417. 
Lansdale, Mark W. 1988. “The Psychology of Personal Information Management." Applied Ergonomics 19(1):55-66.

Levy, Steven. 2011. In the Plex: How Google Thinks, Works, and Shapes Our Lives. New York: Simon and Schuster.

Malone, Thomas W. 1983. “How Do People Organize Their Desks? Implications for the Design of Office Information Systems." ACM Transactions on Office Information Systems (TOIS) 1(1):99-112.

Marchionini, Gary. 1995. Information Seeking in Electronic Environments. Cambridge: Cambridge University Press.

Norman, Donald. 1998. The Invisible Computer. Cambridge, MA: MIT Press.

Norman, Donald. 2010. Living with Complexity. Cambridge, MA: MIT Press.

Teevan, Jaime, Robert Capra, and Manuel Pérez-Quiñones. 2007. “How People Find Personal Information." Pp. 22-34 in Personal Information Management, edited by William Jones and Jaime Teevan. Seattle: University of Washington Press.

Weiser, Mark. 1991. “Computer for the 21st Century." Scientific American, September, 94-104.

\section{SEARCH \\ AND INTERFACES FOR SEARCH}

\section{Ilya Utekhin}

Ilya Utekhin is a professor in the Department of Anthropology at the European University at St. Petersburg. Address for correspondence: ul. Gagarinskaia, 6/1, Saint Petersburg, 191187, Russia.utekhin@yandex.ru.

The development of information technology over the last three decades has revolutionized everyday activities in a domain that can be referred to as personal information management. The personal information environment in which this management takes place consists of both digital and nondigital information artifacts, which a user employs systematically. Interfaces of digital information technology employ analogies for nondigital affordances and nondigital methods of handling information artefacts. Ethnographic studies of nondigital information management have established two poles toward which personal information management strategies tend to gravitate: systematization of documents kept in topically organized folders and keeping documents in chronologically organized piles. Interfaces of digital information technologies provide affordances for both types of strategies. Affordances that make possible dealing with digital information artifacts provide means for finding objects without caring about their location; this is an essentially new feature, unavailable in the nondigital world. Context-sensitive technologies on mobile platforms add opportunities for everyday search such as allowing for finding objects within the information layer of augmented reality and satisfying users' need for services in addition to simply finding information. Everyday search practices have been most affected by technology that analyzes data on users' behavior and displays certain degree of agency while providing personalized recommendations and offering objects that the user did not know about or did not look for.

Keywords: Search; Human-Computer Interaction; Interface; Personal Information Management; Agency 


\section{REFERENCES}

Baeza-Yates, Ricardo, Andrei Z. Broder, and Yoelle Maarek. 2011. “The New Frontier of Web Search Technology: Seven Challenges." Pp. 3-9 in Search Computing: Trends and Developments, edited by Stefano Ceri and Marco Brambilla. New York: Springer.

Berners-Lee, Tim, James Hendler, and Ora Lassila. 2001. “The Semantic Web." Scientific American, May, 28-37.

Broder, Andrei Z. 2002. "A Taxonomy of Web Search." ACM SIGIR Forum 36(2):3-10.

Chipchase, Jan. 2008. "Reducing Illiteracy as a Barrier to Mobile Communication." Pp. 79-89 in Handbook of Mobile Communication Studies, edited by James E. Katz. Cambridge, MA: MIT Press.

Cutrell, Edward, Susan Dumais, and Jaime Teevan. 2006. "Searching to Eliminate Personal Information Management." Communications of ACM 49(1):58-64.

Hillis, Ken, Michael Petit, and Kylie Jarrett. 2013. Google and the Culture of Search. London: Routledge.

Jones, William. 2008. Keeping Found Things Found: The Study and Practice of Personal Information Management. Burlington, MA: Morgan Kaufmann Publishers.

Jones, William, Harry Bruce, and Susan Dumais. 2001. "Keeping Found Things Found on the Web." Pp. 119-126 in Proceedings of the Tenth International Conference on Information and Knowledge Management. Atlanta, GA: ACM.

Jones, William, and Jaime Teevan, eds. 2007. Personal Information Management. Seattle: University of Washington Press.

Jones, William, Abe Wenning, and Harry Bruce. 2014. “How Do People Re-Find Files, Emails and Web Pages?" Pp. 552-564 in Breaking Down Walls: Culture, Context, Computing, edited by Maxi Kindling and Elke Greifeneder. Proceedings of iConference 2014, Berlin, March 4-7, 2014. http:// hdl.handle.net/2142/47417.

Lansdale, Mark W. 1988. “The Psychology of Personal Information Management." Applied Ergonomics 19(1):55-66.

Levy, Steven. 2011. In the Plex: How Google Thinks, Works, and Shapes Our Lives. New York: Simon and Schuster.

Malone, Thomas W. 1983. “How Do People Organize Their Desks? Implications for the Design of Office Information Systems." ACM Transactions on Office Information Systems (TOIS) 1(1):99-112.

Marchionini, Gary. 1995. Information Seeking in Electronic Environments. Cambridge: Cambridge University Press.

Norman, Donald. 1998. The Invisible Computer. Cambridge, MA: MIT Press.

Norman, Donald. 2010. Living with Complexity. Cambridge, MA: MIT Press.

Teevan, Jaime, Robert Capra, and Manuel Pérez-Quiñones. 2007. “How People Find Personal Information." Pp. 22-34 in Personal Information Management, edited by William Jones and Jaime Teevan. Seattle: University of Washington Press.

Utekhin, Il'ia. 2012. “Vzaimodeistvie s 'umnymi veshchami': Vvedenie v problematiku.” Antropologicheskii forum 17:134-156.

Weiser, Mark. 1991. “Computer for the 21st Century." Scientific American, September, 94-104. 